\title{
TRANSMISSION AND PATHOGENESIS OF SEVERE ACUTE RESPIRATORY SYNDROME CORONAVIRUS 2
}

\author{
BEHAILU ASSEFA*
}

Department of Veterinary Microbiology, Immunology and Public health, College of Veterinary Medicine and Agriculture, Addis Ababa University, Bishoftu, Ethiopia. Email: newbaye@gmail.com

Received: 09 September 2020, Revised and Accepted: 11 January 2021

\section{ABSTRACT}

Coronavirus disease 2019 (COVID-19) is a respiratory system disease that results in flu-like symptoms. It is caused by severe acute respiratory syndrome coronavirus 2 (SARS-COV-2) which was initially transmitted from either sea animals, wild animals like bats, or pet animals like dogs, and cats to humans. Since the occurrence of the first human COVID-19 case in China in 2019, the disease uses a trans-human transmission pathway. Its pathogenesis is almost similar to other respiratory diseases.

Keywords: Coronavirus disease 2019, Human, Pathogenesis, Severe acute respiratory syndrome coronavirus 2, Transmission.

(C) 2021 The Authors. Published by Innovare Academic Sciences Pvt Ltd. This is an open access article under the CC BY license (http://creativecommons. org/licenses/by/4.0/) DOI: http://dx.doi.org/10.22159/ijms.2021v9i2.39682. Journal homepage: https://innovareacademics.in/journals/index.php/ijms

\section{INTRODUCTION}

Coronavirus is the already known virus on the globe. It is believed to exist on the globe for many years back. It existed in different forms and different species of animals as well as in the external environment. Some of the forms of this virus have existed since its occurrence in the universe while others were emerged after a time, from the previous forms in different ways. Both the original forms, as well as the emerged forms of coronavirus, have varying degrees of pathogenicity. Some of these different forms of the virus could pass from one species of animal (their primary host) to other susceptible species of animals. Examples of the known zoonotic coronavirus forms are severe acute respiratory syndrome (SARS) corona and Middle-East respiratory syndrome (MERS) corona that could infect human beings passing from animals. Both SARS corona and MERS coronavirus diseases were the major challenges of mankind at a different time in history [1].

Human beings have tried to investigate the root cause of every disease that has happened at a different time in their history in this Universe. Science may not have proof of specific causes of some human challenges. In this case, what is done is logically guessing the occurrence using probabilities and predictions. By the same talking, for the very source of the current coronavirus disease 2019 (COVID-19) outbreak, what science has brought is guessing. This disease is widely taught to originate from animals. The consumption of aquatic animals, wild animals (bats), or domestic animals such as dogs and cats which are not usually known as animals for human consumption were believed to be a primary source of the current pandemic disease, COVID-19. This was the case in China which was the very first spot for the disease to occur in the human. Whether there existed another transmission mechanism of this disease from animals to humans, in addition to the consumption of animal products, was not yet validated. What is known at present is that once the disease steps its foot in humans from its source in animals, it freely moves across the human population of the globe [2].

\section{TRANSMISSION}

Even though there is a debate on the primary source of COVID-19 for the first human case, now the source for the succeeding human cases is known. These sources could be either the previously infected human or a contaminated environment, particularly materials contaminated by human's infective droplets. The virus in an infected human or on contaminated material may get access to another human through direct contact with infected human or contaminated materials [3].

If the infected human sneeze or coughs or just exhale at a closer distance (usually at a 2-2-m radius) from a susceptible human, the active virus droplets usually get into the respiratory system of the second person. Introduction of the virus through the eye could also be possible. In case there were no human receptors during the sneezing or coughing of the infected human, the discharged virus may stay on any material nearby. There are cases in which these secondary sources become infective to humans. A human may handle these contaminated materials or if it is a foodstuff, may eat them before being properly sterilized. Then, by the unsterilized hand, he may touch his eyes, nose, mouth, or ears. By so doing, he let the virus gain access to the internal body where it can multiply and then cause infection [4].

The passage of the virus from one infected human being to another is now the major passageway against which every control effort is directing. Due to this priority, studies on the passage of the virus from animals to humans do not get much attention. Besides the transmission of the virus from an infected human to another human being, there may be a continual passage of the virus from the primary source animals to human as the previous practices of consuming the suspected animals continues at present particularly in the source country China [5].

There is no strong evidence about the existence of reservoirs and reinfection cases in COVID-19. Whatever the case, it is better to suspect such cases and gives due attention in addition to working on the trans-human transmission of the virus. Among human transmission mechanisms by themselves should be exhaustively validated. Because sometimes cases in which it is difficult to trace how the infection could occur happened. The potential of the virus to be viable in different environments until it catches the human host and the distance it can move in the air at the time of discharge from an infected host and even from where it precipitates on some hard materials is not well known [6].

\section{PATHOGENESIS}

The pathogenesis of SARS-coronavirus 2 (COV-2) is not well identified. This may also potentiate the rapid passage of the virus among the human population of the globe. Because, not knowing the pathogenesis of a 
disease could hinder its early and effective control. The virus has different routes of entry to humans. The identified routes of entry include nasal, oral, conjunctival, and through the ears. Either direct passage from an infected source or hand-induced source could be the main source of the virus for these routes of entry. A hand is a major tool for the passage of this virus from an infected source to others. Due to this, one's own hands become a danger and a fear, as equal as an infected person [7].

Once the SARS-COV-2 gains access to an internal body of humans through either of the above routes, it usually prefers to dwell in the respiratory systems. The respiratory system is the predilection site for this virus. The virus could affect humans and can result in death when it binds to any part of the respiratory system. Binding to and multiplying in the cells of the respiratory system, the virus interferes with the normal breathing system of the host. Difficult breathing and hypoxia are the primary cause of death to COVID-19. Besides, the SARS-COV-2 is an immunosuppressant that decreases the immunity (resistance) of an individual to any pathogen just like any other virus. Then the reduced immunity could further liable an individual to other disease complications [8].

The incubation period of the SARS-COV-2 virus ranges from 2 days to 2 weeks. The incubation period varies depending on the immune status of the host, age of an infected individual, presence or absence of other health complications, physiological state of female individuals, and a dose and route of entry of the virus. In some cases, the disease is symptomless while in others it is clinical which is manifested by flu-like symptoms. There is no pathognomonic sign for COVID-19. An infected individual under any one of the above disease states could shade an infective virus and become a danger to pass the disease to others [9].

If the person caught by SARS-COV-2 has already got other health complications such as heart problems, diabetes, kidney problem, and other respiratory problems such as asthma and any inflammation to respiratory parts and/or respiratory failure due to smoking, the case could be much aggravated. There is no evidence of whether the SARSCOV-2 enters the body through other routes than the natural opening of the body. Still, there is no evidence whether the SARS-COV-2 could result in death due to its multiplication in other organ systems in addition to death due to respiratory complications [10]

\section{CONCLUSION AND RECOMMENDATION}

A lot is left to be investigated about the transmission mechanism of SARS-COV-2 from their very source in any of the life forms or abiotic environments to humans. Besides, the pathogenesis of this virus needs to be further investigated whether any other organ system is involved and could equally be responsible for the human deaths to COVID-19 as of the respiratory system.
In the meantime, intensively applying control mechanisms to decrease the passage of COVID-19 among the human population by interrupting the transmission pathways is mandatory. It is recommended to apply containment measures by limiting the virus within the infected individuals using methods such as isolating infected individuals by a strict quarantine system, using face masks, gloves, and protective clothes, or at least covering the nose and mouth at sneezing or coughing by an elbow. The last recommended control measures to be taken are protecting the uninfected but exposed individuals by avoiding contact with infected individuals by letting them stay at home, or if not possible to stay home, keeping at least a 2-m distance from any other individuals. The use of face masks, washing hands after every handling of potential virus source and avoiding touching nose, mouth, eye, and ear by hand before washing are all recommended practices to be followed to control the COVID-19 pandemic.

\section{REFERENCES}

1. Mackenzie JS, Smith DW. COVID-19: A novel zoonotic disease caused by a coronavirus from China: What we know and what we don't. Microbiol Aust 2020;1:MA20013.

2. Institute of Medicine (US) Forum on Microbial Threats. Microbial evolution and co-adaptation: A tribute to the life and scientific legacies of joshua lederberg: Workshop summary. In: 5 Infectious Disease Emergence: Past, Present, and Future. Washington, DC: National Academies Press; 2009.

3. World Health Organization. Coronavirus Disease 2019 (COVID-19). Situation Report No. 94. Data as Received by World Health Organization from National Authorities by 10:00 CEST; 2020.

4. World Health Organization. How World Health Organization is Working to Track Down the Animal Reservoir of the SARS-CoV-2 Virus 2020; 2020 b.

5. World Health Organization. SARS-CoV-2 Mink-Associated Variant Strain-Denmark, Disease Outbreak News; 2020.

6. Cash-Goldwasser S, Kardooni S, Cobb L, Bochner A, Bradford E, Shahpar C. Weekly COVID-19 Science Review August 22-28 2020, Resolve to Save Lives; 2020. Available from: https://www. preventepidemics.org/coronavirus/weekly-science-review.

7. Zheng J. SARS-CoV-2: An emerging Coronavirus that causes a global threat. Int J Biol Sci 2020;16:1678-85.

8. World Health Organization. Coronavirus Disease 2019 (COVID-19). Situation Report No. 32, Data as Reported by 21 February 2020; 2020.

9. National Library of Medicine. National Library of Medicine, or "Vancouver Style" (International Committee of Medical Journal Editors): DynaMed, 1995-Record No. T1579903929505. Ipswich, MA: EBSCO Information Services; 2020. Available from: https://www. dynamed.com/topics/dmp an t1579903929505. [Last accessed on 2020 Aug 01].

10. Johansen MD, Irving A, Montagutelli X, Tate MD, Rudloff I, Nold MF. Animal and translational models of SARS-CoV-2 infection and COVID-19. Mucosal Immunol 2020;13:877-91. 